\title{
Computer simulations of the random barrier model
}

\author{
Thomas B. Schroder and Jeppe C. Dyre* \\ Department of Mathematics and Physics (IMFUFA), Roskilde University, Postbox 260, \\ DK-4000, Roskilde, Denmark.E-mail: dyre@ruc.dk
}

Received 19th December 2001, Accepted 11th March 2002

First published as an Advance Article on the web 5th June 2002

\begin{abstract}
A brief review of experimental facts regarding ac electronic and ionic conduction in disordered solids is given followed by a discussion of what is perhaps the simplest realistic model, the random barrier model (symmetric hopping model). Results from large scale computer simulations are presented, focusing on universality of the ac response in the extreme disorder limit. Finally, some important unsolved problems relating to hopping models for ac conduction are listed.
\end{abstract}

\section{Introduction}

This paper discusses a simple model for ac conduction in disordered solids. First, we shall briefly review the experimental facts to be explained. ${ }^{1-4}$

\section{A. The experimental situation}

In the present context "disordered solids" are non-metals which are not perfect crystals. In many cases the solids in question are glassy or amorphous, but in some cases defective crystals or polycrystalline samples are considered. A very interesting point is the fact that electronic and ionic conduction in disordered solids are so similar that they cannot be distinguished by means of bulk ac measurements, a discovery of the 1970's. ${ }^{5-9}$ This implies that relevant models must involve only few and general assumptions.

Ac conduction is measured at frequencies much below phonon frequencies $\left(\omega \ll 10^{13} \mathrm{~s}^{-1}\right)$ [in this paper the term "frequency" always means "angular frequency"]. Nowadays the ac conductivity $\sigma(\omega)$ is routinely measured down to frequencies corresponding to periods of the applied electric field longer than $1000 \mathrm{~s}$. The quantity $\sigma(\omega)$ is in general complex; any non-zero imaginary part signals a phase difference between the sinusoidal electric field and the resulting sinusoidal current.

As mentioned, the ac conductivities of quite diverse disordered solids are surprisingly similar. This fact is referred to as "ac universality"., $3,10,11$ On the one hand it means that a simple, universally applicable model is likely to exist, but, on the other hand, it also means that little is to be learned from "quick and dirty" ac measurements. Of course, experiments on diverse solids do not give exactly the same $\sigma(\omega)$, so this quantity does contain some sample specific information. Nevertheless, the observed universality (perhaps more correctly: "quasi-universality") ${ }^{12}$ makes it hard to learn specifics about the conduction process solely from $\sigma(\omega)$.

There are numerous papers in the literature presenting ac data for disordered solids like ionic conductive glasses, ${ }^{13}$ amorphous semiconductors, ${ }^{14}$ polymers, ${ }^{15}$ etc. The experimental findings may be summarized as follows: ${ }^{4}$

(i) The real part of the ac conductivity increases with frequency; the imaginary part is non-negative. At high frequencies $\sigma^{\prime}(\omega)$ follows an apparent power law,

$$
\sigma^{\prime}(\omega) \propto \omega^{n}
$$

with $n$ increasing weakly with frequency. At low frequencies there is a gradual transition to frequency independent conductivity. In a $\log -\log$ plot the ac conductivity is much less temperature dependent than the dc conductivity. The ac exponent $n$ is between 0.6 and 1.0. In a fixed frequency range $n$ increases as temperature $T$ decreases; $n \rightarrow 1.0$ for $T \rightarrow 0$ and when $n$ is close to $1.0 \sigma^{\prime}(\omega)$ is almost temperature independent. When there is no measurable dc conductivity $n$ is close to 1.0 .

(ii) The ac conductivity obeys time-temperature superposition, i.e., the shape of $\sigma^{\prime}(\omega)$ in a $\log -\log$ plot is temperature independent. This makes it possible to construct a master curve from measurements at different temperatures. Time-temperature superposition applies also for $\sigma^{\prime \prime}(\omega)$. The shape of the master curve is roughly the same for all disordered solids.

(iii) Whenever $\sigma(0)$ is measurable there is a dielectric loss peak [the dielectric loss is the imaginary part of $\varepsilon(\omega)$ we follow the convention in the field by defining $\varepsilon(\omega) \equiv \varepsilon_{\infty}+[\sigma(\omega)-\sigma(0)] /\left(\mathrm{i} \omega \varepsilon_{0}\right)$ where $\sigma(\omega)$ is the hopping contribution to the conductivity]. The onset of ac conduction takes place around the dielectric loss peak frequency, $\omega_{m}$. The Barton-Nakajima-Namikawa (BNN) relation is obeyed: $\sigma(0)=p \Delta \varepsilon \varepsilon_{0} \omega_{m}$, where $p$ is a numerical constant of order one and $\Delta \varepsilon$ is the dielectric loss strength $(\Delta \varepsilon \equiv \varepsilon(0)-\varepsilon(\infty))$. The latter is much less temperature dependent than $\omega_{m}$ or $\sigma(0)$, so the BNN relation implies a rough proportionality $\sigma(0) \sim \omega_{m}$. In most cases $\sigma(0)$ and $\omega_{m}$ are Arrhenius temperature dependent (with the same activation energy).

\section{B. Two models for ac conduction in disordered solids}

There are many approaches to the description of ac phenomena. Many "models" are by definition mathematical expressions for $\sigma(\omega)$ which are derived by plausible arguments; in fact for many scientists a "model" is identified with the mathematical expression arrived at. We shall use the term "model" in a different sense, similar to that of, e.g., the Ising model for ferromagnetism: A model is an attempt to identify the essential features of the phenomenon in question, leading to an exact mathematical formulation. Usually, the model cannot be solved rigorously, but is instead studied by means of computer simulations and/or analytical approximations.

In this section we briefly discuss two models. ${ }^{4}$ The first is the so-called "hopping model." This model, which describes the random motion of non-interacting particles, is the subject of 
this paper where a particularly simple instance of hopping is studied. Consider hopping of particles in the absence of an external electric field. For simplicity the particles can sit only on the sites of a simple cubic lattice. The solid disorder is reflected in spatially randomly varying jump probabilities (usually referred to as jump frequencies). In the simplest case the particles are non-interacting, in more realistic cases there is self-exclusion at each site and Coulomb interactions. We shall mainly be concerned with the simplest version of the model, i.e., the case where particles are non-interacting. Because the particles move independently it is only necessary to consider the motion of one single particle. It may be shown that in any hopping model $\sigma^{\prime}(\omega)$ is an increasing function of frequency. ${ }^{16,17}$ Moreover, when the model involves jump frequencies covering several orders of magnitude $\sigma^{\prime}(\omega)$ also increases several orders of magnitude, as in experiment. In an external field the linear response is calculated by reference to the fluctuation-dissipation theorem, which shows how to calculate $\sigma(\omega)$ from a knowledge of the equilibrium dynamics. Thus, from now on we shall only discuss the zero-field case.

A second model for ac conduction in disordered solids which is physically quite different from the hopping model, is the macroscopic model. ${ }^{18}$ This model regards the solid as a spatially random mixture of phases with differing conductivities. Each phase has a frequency-independent conductivity, but because of the mixing there is frequency dependence of the overall conductivity. The macroscopic model leads to a linear differential equation with randomly varying coefficients, much like the master equation for the hopping model discussed in the next section. Because the model is based on Maxwell's equations, it takes into account all Coulomb interactions between charge carriers, as well as between charge carriers and immobile charges. In particular, this implies that in nonzero fields the macroscopic model has a spatially varying electric field (while the hopping model assumes a spatially homogeneous field because all interactions, including Coulomb interactions, are ignored). Nevertheless, the two models are mathematically similar and so are their predictions. ${ }^{4}$ The main weakness of the macroscopic model is that it cannot describe solids that are disordered on the atomic scale, as is most likely the case for many disordered solids.

\section{The random barrier model}

Before presenting results for $\sigma(\omega)$ from computer simulations of the random barrier model we first define hopping models generally and discuss how percolation determines the activation energy of the dc conductivity.

\section{A. Hopping models and the role of percolation}

Hopping models give a stochastic description of charge carrier motion. ${ }^{16,17,19-23}$ A hopping model is defined by specifying the allowed transitions and their transition rates. In most cases, an allowed transition involves the displacement of just one particle, but it is also possible to include multiparticle jumps. The single particle jump model, however, is already quite complex. Further simplifications are arrived at by ignoring interactions and by assuming that the set of allowed charge carrier positions defines a regular lattice. If Coulomb interactions are ignored (both between charge carriers and between charge carriers and fixed "counter-ions") a so-called lattice gas model is arrived at. The only interaction left here is self-exclusion, i.e., the fact that there is room for just one charge carrier at each site. If self-exclusion is also ignored, one has completely noninteracting particles; in this case all relevant information is known once the motion of single particles is understood. We shall consider a model of this type.
By assumption the particles can take positions only on a simple cubic lattice in $d$ dimensions. The basic quantity characterizing such an ensemble of particles is the probability $P_{s}$ to be at site $s$. If $\Gamma\left(s \rightarrow s^{\prime}\right)$ is the probability per unit time for jumps from site $s$ to site $s^{\prime}$, the "equation of motion" is the wellknown master equation ${ }^{19-21,24}$

$$
\frac{\mathrm{d}}{\mathrm{d} t} P_{s}=\sum_{s^{\prime}}\left(\Gamma\left(s^{\prime} \rightarrow s\right) P_{s^{\prime}}-\Gamma\left(s \rightarrow s^{\prime}\right) P_{s}\right) .
$$

Eqn. (2.2) determines the equilibrium dynamics. From equilibrium fluctuations the velocity autocorrelation function $\langle v(0) v(t)\rangle$ may be calculated where $v$ is the projection of the velocity in a fixed direction. This quantity determines the frequency-dependent diffusion constant $D(\omega)$ by ${ }^{17,25}$

$$
D(\omega)=\int_{0}^{\infty}\langle v(0) v(t)\rangle \mathrm{e}^{-\mathrm{i} \omega t} \mathrm{~d} t .
$$

In turn, this quantity determines $\sigma(\omega)$ by the fluctuation-dissipation theorem, ${ }^{26} \sigma(\omega)=n q^{2} D(\omega) / k_{\mathrm{B}} T$, where $n$ is particle density and $q$ the carrier charge. In this paper, as often in theoretical discussions of hopping models, ${ }^{21}$ a unit system is used in which $\sigma(\omega)=D(\omega)$ and where moreover both quantities are normalized such that on a lattice with uniform jump frequency $\Gamma$ one has $\sigma(\omega)=D(\omega)=\Gamma$ (in the uniform case the conductivity is frequency independent). We define the dimensionless frequency-dependent conductivity by $\tilde{\sigma}(\omega)=\sigma(\omega) / \sigma(0)$.

The random barrier model is defined by a special case of eqn. (2.2), namely that corresponding to the case where all sites have equal energy. ${ }^{27}$ Fig. 1 illustrates the random energy landscape of this model in one dimension. Whenever site energies are equal, by the principle of detailed balance ${ }^{24}$ (a consequency of time-reversal invariance) the jump rates are symmetric: $\Gamma\left(s \rightarrow s^{\prime}\right)=\Gamma\left(s^{\prime} \rightarrow s\right)$. If this quantity is denoted by $\Gamma\left(s, s^{\prime}\right)$, eqn. (2.2) reduces to

$$
\frac{\mathrm{d}}{\mathrm{d} t} P_{s}=\sum_{s^{\prime}} \Gamma\left(s, s^{\prime}\right)\left(P_{s^{\prime}}-P_{s}\right) .
$$

We shall assume that the jump rates are given by an energy barrier $E: \Gamma=\Gamma_{0} \exp (-\beta E)\left(\Gamma_{0}=1\right.$ in simulations), where $\beta=1 / k_{\mathrm{B}} T$ ( $\beta$ is the inverse wave function size if quantum mechanical tunnelling is considered). The model is now completely defined in terms of the energy barrier probability distribution, $p(E)$. Our main focus is the case when $\beta \rightarrow \infty$. This is termed the extreme disorder limit, because in this limit jump rates vary many orders of magnitude.

The random barrier model describes a highly inhomogeneous situation. This is illustrated in two dimensions in Fig. 2, which shows the probability distribution after some time for one particle starting in the centre. The figure contrasts (a) the high temperature limit where all jump rates become equal (homogeneous situation) to (b) the low temperature, highly inhomogeneous case. In the latter case at intermediate frequencies not all sites situated symmetrically around the

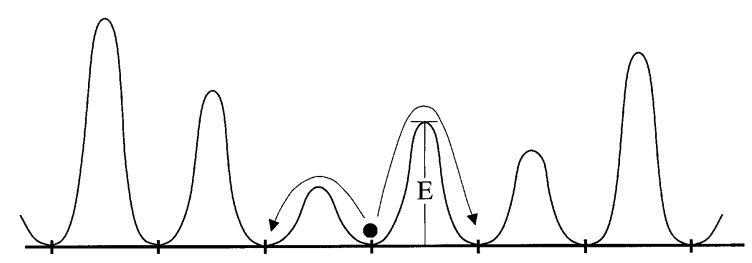

Fig. 1 One dimensional version of the energy landscape defining the random barrier model. The minima give the allowed particle positions. All minima have equal energy, implying that all positions are equally likely. The barrier heights vary randomly according to a probability distribution $p(E)$. The jump rate for jumps between neighbouring sites, $\Gamma$, is given by $\Gamma=\Gamma_{0} \exp (-\beta E)$, where $\beta=1 / k_{\mathrm{B}} T$. 
(a)

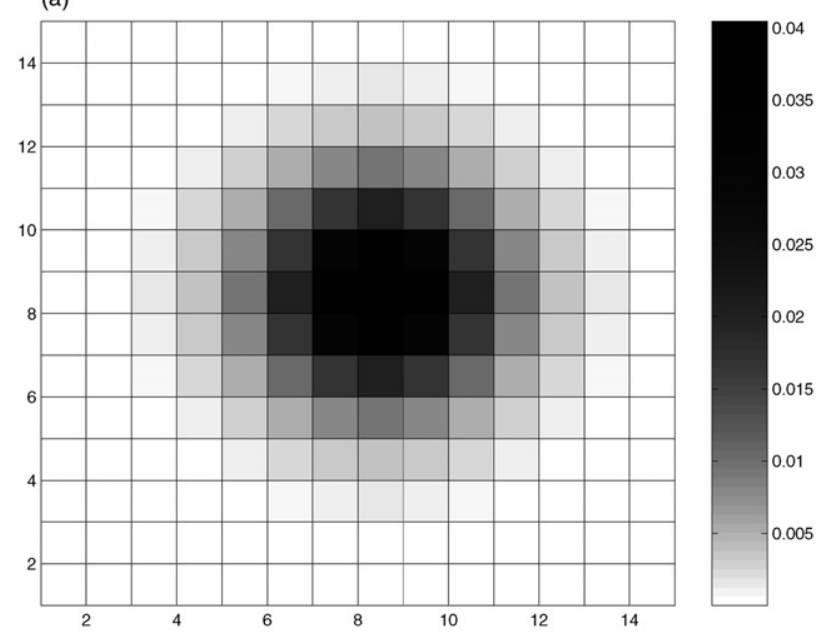

(b)

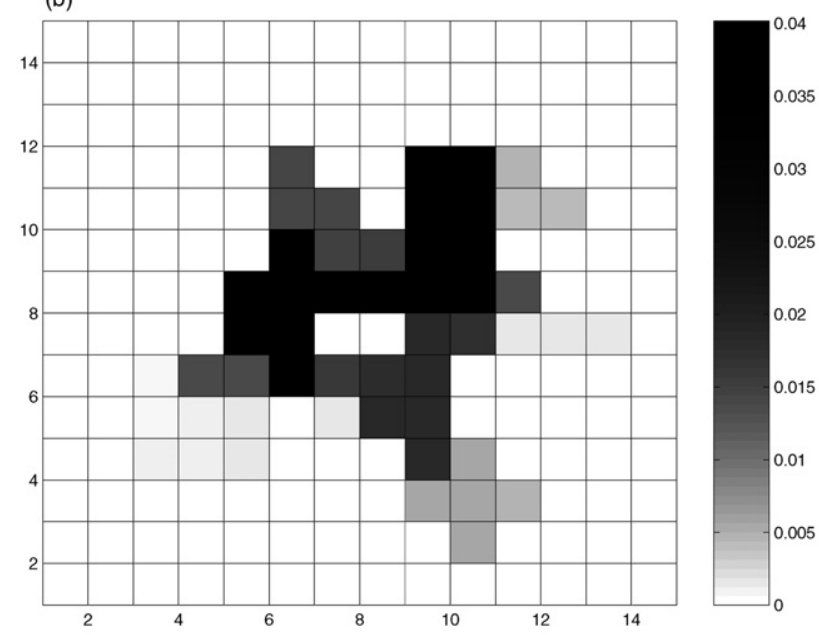

Fig. 2 Probabilities for the 2-dimensional random barrier model with box distribution of activation energies some time after starting in the centre. Each site is represented by a square which is colored a shade of grey according to the value of the probability at the given site. (a) Shows the situation corresponding to no disorder: $\beta=0$ where $\beta$ is inverse temperature; (b) shows the case $\beta=40 .^{28}$ In the latter case, some sites asymmetrically distributed around the centre are more likely than others, reflecting the disorder. Note, however, that this applies only at intermediate times; as $t \rightarrow \infty$ all sites become equally probable.

centre are visited with same probability; however as time goes to infinity this will eventually be the case.

In the next section we shall investigate the ac conductivity of the random barrier model. Before that, let us consider the temperature dependence of the dc conductivity. A priori, because a broad range of barrier energies are involved in dc transport, one would not expect any simple temperature dependence of $\sigma(0)$. Actually, in both experiment and computer simulations one finds a simple Arrhenius temperature dependence of $\sigma(0){ }^{6,28}$ The reason for this has been well understood for 30 years: ${ }^{20,29-31}$ The Arrhenius behaviour is a consequence of the fact that percolation "rules" dc conduction when the disorder is sufficiently strong. Briefly, percolation arises as follows. Suppose lattice links are marked in order of increasing barrier energy. First, the marked links are isolated from one another and form only small clusters. As a definite fraction of links $p_{\mathrm{c}}$ is reached, the (link) "percolation threshold", an infinite cluster forms. Particle motion on the percolation cluster is optimal in the sense that large barriers are avoided as best possible. The largest barrier on the percolation cluster, $E_{\mathrm{c}}$, determines the bottleneck for dc conduction. Consequently, the dc conductivity activation energy $E_{\mathrm{c}}$ is determined from

$$
\int_{0}^{E_{\mathrm{c}}} p(E) \mathrm{d} E=p_{\mathrm{c}} .
$$

Fig. 3 shows the dependence of $\sigma(0)$ on inverse temperature for the box probability distribution of barriers (uniform distribution between zero and $E_{0}$-we put $E_{0}=1$ which corresponds to measuring energy in units of $E_{0}$ and temperature in units of $E_{0} / k_{\mathrm{B}}$ ). For each $\beta$ the results were averaged over 100 different $N \times N \times N$ samples, with $N$ varying from 14 $(\beta=20)$ to $N=96(\beta=320)$. By varying $N$ for each $\beta$ it was estimated that the relative error in the results due to finite size effects is at most $3 \%$. At each $\beta$ energy barriers larger than $E_{\mathrm{c}}+k / \beta$ were removed from the sample. By varying $k$ it was estimated the the relative error due to this procedure (with $k=6.4)$ is less than $1 \%$. Since $p_{\mathrm{c}}=0.2488$ and $E_{\mathrm{c}}=p_{\mathrm{c}}$ for this distribution, one expects $\sigma(0)$ to (numerically) have this slope when $\ln (\sigma(0))$ is plotted as function of $\beta$. The inset shows that the (weakly) temperature-dependent prefactor for high values of $\beta$ varies as $\beta^{-y}$. In three dimensions the exponent $y$ is predicted ${ }^{32}$ to be equal to the correlation length exponent $\nu$ of percolation theory $(\nu \approx 0.88),{ }^{33}$ in agreement with what we find here. This is utilized in the main figure to determine the slope, which is indeed that predicted from percolation theory.

\section{B. Computer simulations of ac conductivity}

The numerical results presented in this paper were arrived at using the velocity auto-correlation (VAC) method described in ref. 28. This method is based on solving the master equation and thus does not suffer from the noise inherent in Monte Carlo methods. To avoid numerical problems the smallest energy barriers were raised to a frequency-dependent minimum value, ensuring that all jump rates are less than $\omega / \delta$ where $\delta$ is a small number (note the dependence on $\omega$ - this modification only has effect at very small frequencies). By

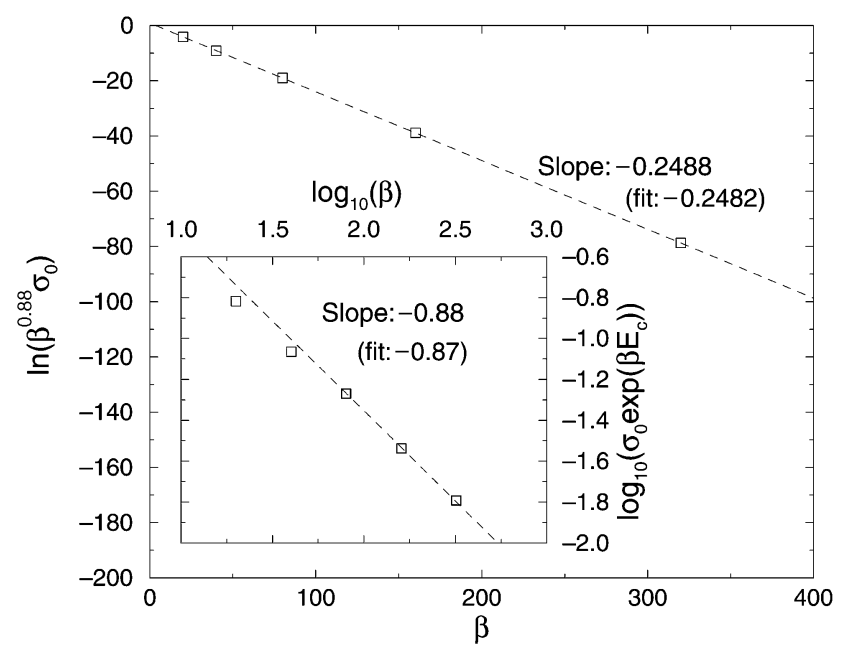

Fig. $3 \beta$-dependence of the dc conductivity, $\sigma_{0}$, in the three dimensional random barrier model with box distribution of energy barriers: $p(E)=1 / E_{0}\left(0 \leqslant E \leqslant E_{0}\right.$; putting $E_{0}=1$ defines the unit of temperature). The unit system used is that where conductivity has the same unit as jump frequency and the jump frequency prefactor is set to one. In the extreme disorder limit (high $\beta$ 's) the predicted behavior is: $\sigma_{0} \propto \beta^{-y} \exp \left(-\beta E_{\mathrm{c}}\right.$ ), where $y=0.88$ (see text) and $E_{\mathrm{c}}=0.2488$. The main figureshows $\ln \left(\beta^{y} \sigma_{0}\right)(y=0.88)$ versus $\beta$, with the dashed line having the predicted slope, $-E_{\mathrm{c}}=-0.2488$. Fitting a straight line to the data in this plot gives a slope of $-0.2482 \pm 0.0003$. The inset shows $\log _{10}\left(\sigma_{0} \exp \left(\beta E_{\mathrm{c}}\right)\right)$ with the predicted $E_{\mathrm{c}}=0.2488$ versus $\log _{10}(\beta)$, with a straight line having the slope $-y=-0.88$. The predicted line is seen to fit the data reasonably well for $\beta \geqslant 80$. Fitting a straight line to the data for $\beta \geqslant 80$ gives a slope $-0.87 \pm 0.01$. 
varying $\delta$ it was estimated that the relative error due to this modification (with $\delta=10^{-14}$ ) is of the order $0.01 \%$.

Ac data taken at different temperatures may be scaled to one single master curve if time-temperature superposition applies. As mentioned, this is found in experiments. As we shall demonstrate shortly this is also found in computer simulations. The conductivity is scaled by dividing out the de conductivity, thus using the dimensionless conductivity $\tilde{\sigma}$ already defined. But how is frequency to be scaled? It has recently been shown mathematically that under reasonable analyticity assumptions there is only one way to scale frequency, ${ }^{34}$ namely to define the dimensionless frequency by $\tilde{\omega} \equiv \omega \Delta \varepsilon \varepsilon_{0} / \sigma(0)$. It is important to note that the dielectric loss $\Delta \varepsilon$ is only weakly temperature dependent compared to the dc conductivity. Nevertheless, it is of interest to determine the temperature dependence of this quantity. This is done in Fig. 4, where it is found that $\Delta \varepsilon \propto \beta^{1.37}$. The diffusion cluster approximation (DCA) (eqn. (2.6) below, $)^{34}$ predicts a similar temperature dependence, albeit with exponent $1.48 .^{17}$

Fig. 5 gives some examples of our results from computer simulations; further results may be found in ref. 28. Fig. 5(a) and (b) give results for the real part of the ac conductivity for the box distribution of energy barriers. These figures show the same data: In (a) "raw" data are given, in (b) the corresponding scaled quantities are used. Clearly, as $\beta \rightarrow \infty$ the data converge to one universal curve. Fig. 5(c) presents scaled data for the exponential barrier distribution. The picture is the same as for the box distribution. We have tested a number of other distributions, always finding the same picture.

What causes ac universality? There have been speculations on this for several years in the literature. Most authors seem to agree that ac universality is somehow an effect of the underlying percolation which thus controls not only dc, but also ac conduction in the extreme disorder limit. Until now, however, most authors proposed approximate analytical expressions for the ac conductivity coming from considerations involving cluster size statistics close to the percolation threshold. We take a radically different viewpoint: Finite clusters isolated from the infinite percolation cluster are unimportant at frequencies where ac universality applies. This point of view was detailed in ref. 4 .

In Fig. 6 the imaginary part of the ac conductivity for the box distribution is presented. Fig. 6(a) gives the raw data; note that zero on the log-frequency scale is really the very high fre-

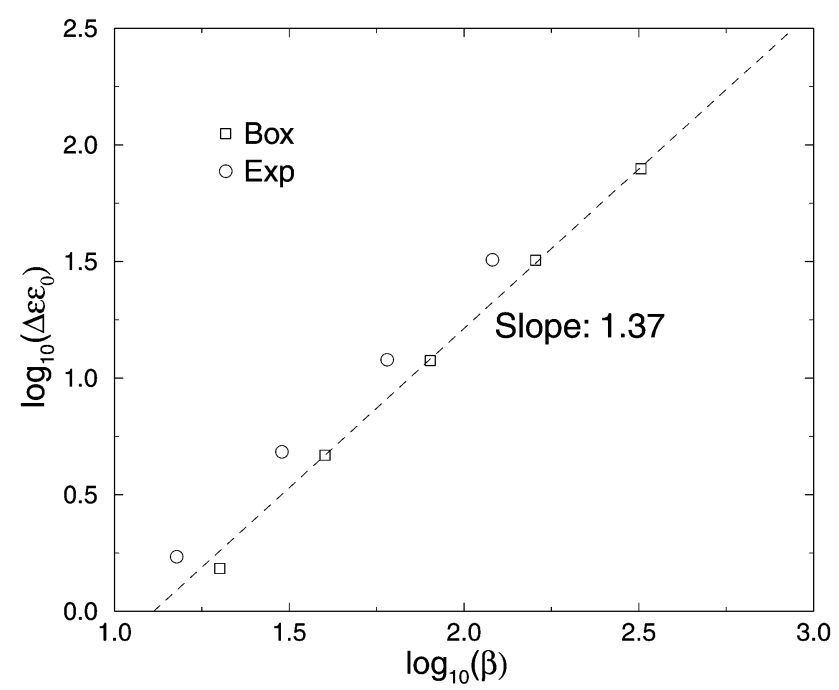

Fig. $4 \log _{10}\left(\Delta \varepsilon \varepsilon_{0}\right)$ versus $\log _{10}(\beta)$ for the box distribution (squares) and the exponential distribution $\left(p(E)=\exp \left(-E / E_{0}\right) / E_{0}, 0 \leqslant E \leqslant \infty\right.$, $\infty$, circles; putting $E_{0}=1$ defines the temperature unit). The straight line is fitted to the data for the box distribution for $\beta \geqslant 40$, corresponding to the expression $\Delta \varepsilon \varepsilon_{0} \propto \beta^{\gamma}$, with $\gamma=1.37$.
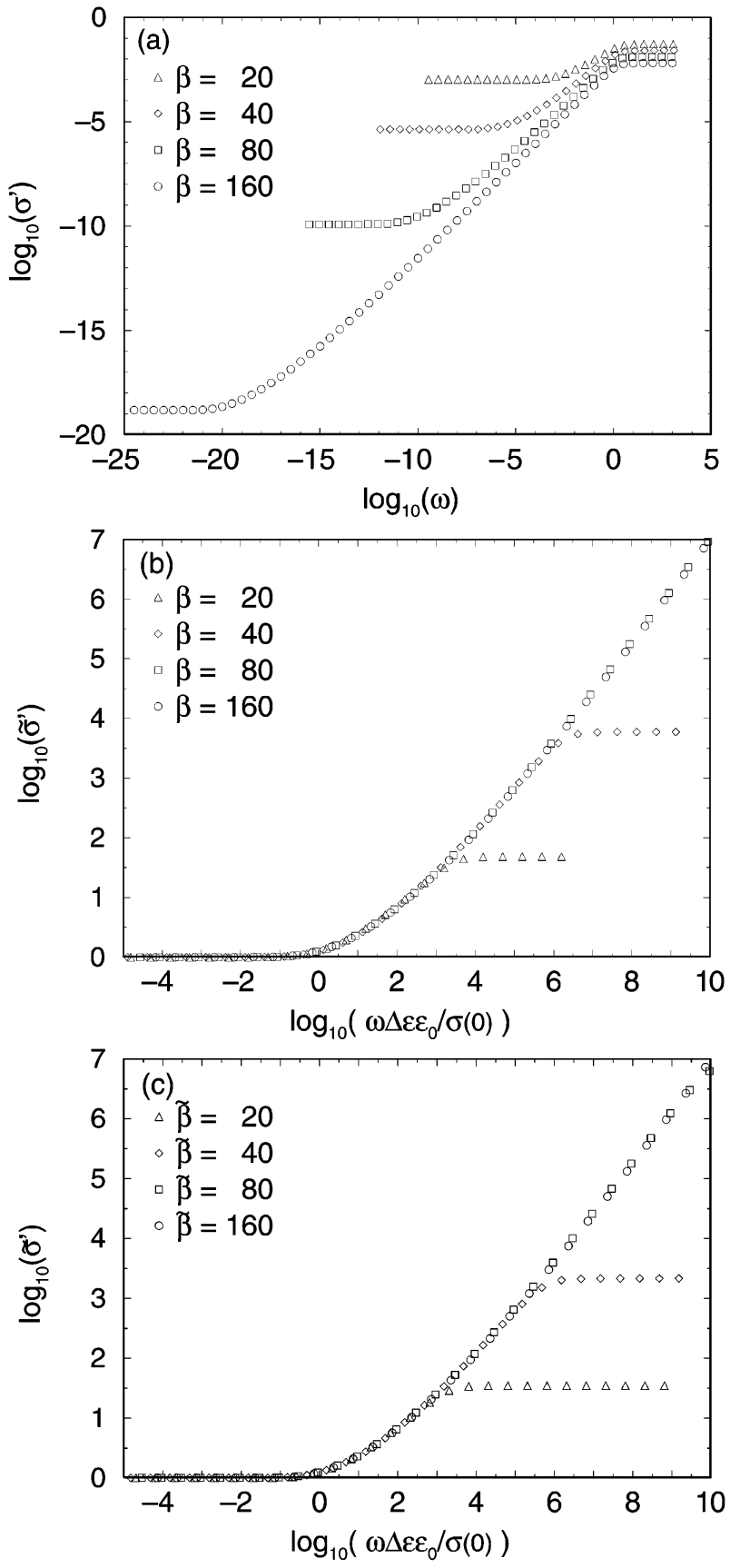

Fig. 5 Results for the real part of the frequency-dependent conductivity, $\sigma^{\prime}(\omega)$. (a) Gives $\sigma^{\prime}(\omega)$ for the box distribution without any scaling applied in the unit system where conductivity has the same unit as frequency and the jump frequency prefactor $\Gamma_{0}$ is one. (b) Shows same data as in (a), but scaled as discussed in the text. Convergence to a "master curve" is evident. At a (scaled) frequency depending on $\beta$ the data change abruptly from the master curve to a frequency independent conductivity (this limit corresponds to time scales so short that the particle does not jump more than once). (c) Shows same as in (b) but now for the exponential distribution of energy barriers $\left(p(E)=\exp \left(-E / E_{0}\right) / E_{0} ; \tilde{\beta} \equiv \beta / p\left(E_{c}\right)\right)$. The same convergence as in (b) is seen, except that the frequency where the data deviate from the master curve is (slightly) different.

quency above which the conductivity becomes frequency independent (compare Fig. 5(a)). Fig. 6 (b) gives the scaled data. Again we find that the scaled conductivity converges to a temperature-independent curve. Note, however, that the convergence is somewhat slower than for the real part. This has important consequences for experiment: The imaginary part of the ac conductivity contains more system specific information than the real part. 

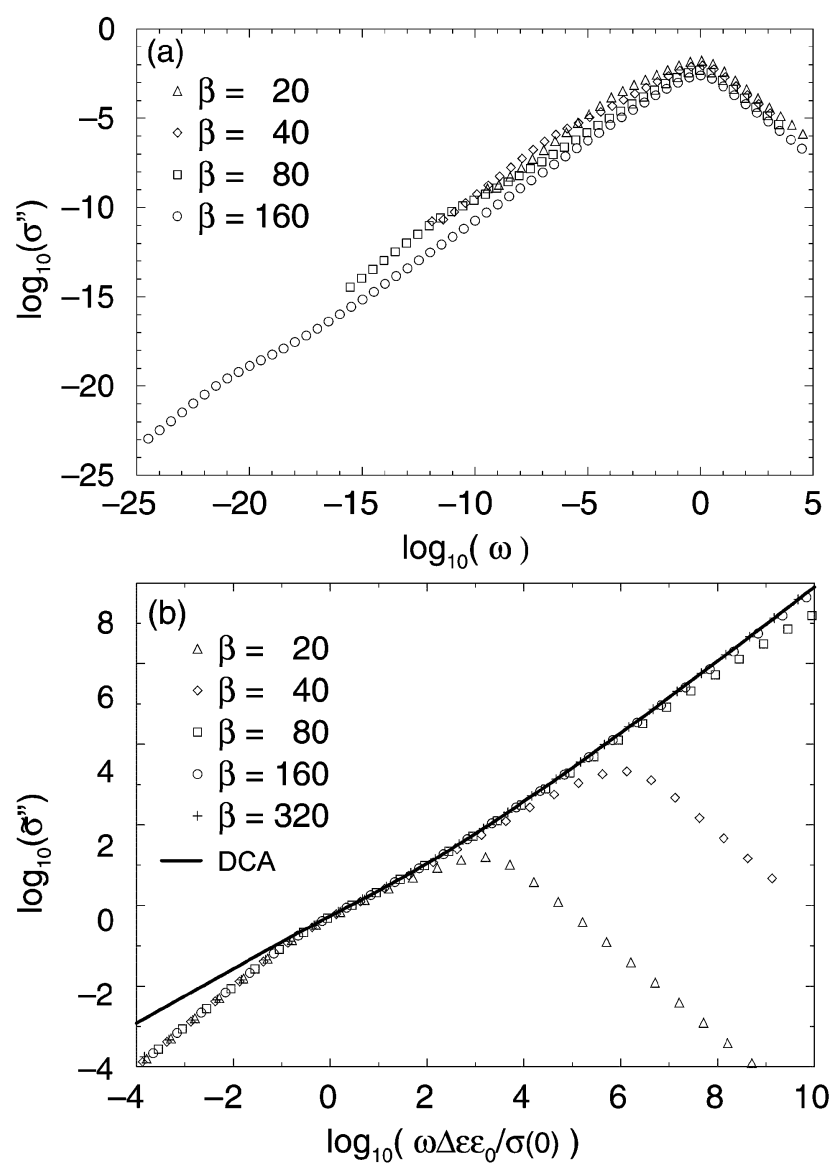

Fig. 6 Results for the imaginary part of the frequency dependent conductivity, $\sigma^{\prime \prime}(\omega)$. (a) Shows $\sigma^{\prime \prime}(\omega)$ for the box distribution without any scaling applied. (b) shows same data as in (a), but scaled as discussed in the text. As is the case for the real part (Fig. 5), the data converge to a master curve, but the convergence is slower. The full line is the diffusion cluster approximation (DCA) [see Section II.C below] empirically scaled by a factor $10^{0.33}$.

\section{Analytical approximation to the universal ac conductivity}

The best available analytical approximation to the universal ac conductivity is the diffusion cluster approximation (DCA).,34 According to the DCA the universal ac conductivity is given as the solution to the following equation

$$
\ln \tilde{\sigma}=\left(\frac{\mathrm{i} \tilde{\omega}}{\tilde{\sigma}}\right)^{d_{0} / 2},
$$

where $d_{0}$ is a suitable exponent. Our simulations in three dimensions are well fitted by DCA with $d_{0}=1.35$. For future reference we give an accurate numerical approximation to the DCA with $d_{0}=1.35$ :

$$
\tilde{\sigma}^{\prime}=1+0.477(\tilde{\omega})^{0.571}+0.026(\tilde{\omega})^{0.872}
$$

This approximation for the real part is accurate on the log scale within 0.01 for the range of scaled frequencies represented in Fig. 7.

The good fit of the DCA with $d_{0}=1.35$ to the real part of simulations is clear from Fig. 7 which shows data for five different barrier distributions. Not visible from this figure are disagreements between DCA (full curve) and simulations found at very low frequencies (compare Fig. 6b): For $\omega \rightarrow 0$ DCA predicts $\tilde{\sigma}-1 \propto(\mathrm{i} \tilde{\omega})^{d_{0} / 2}$ whereas simulations show the result expected from analyticity, $\tilde{\sigma}-1 \propto \mathrm{i} \tilde{\omega}$. Physically, this disagreement is related to the particle at long time moving further than the correlation length of the "fat" percolation cluster. ${ }^{4}$ Also shown in Fig. 7 is the prediction of the effective medium

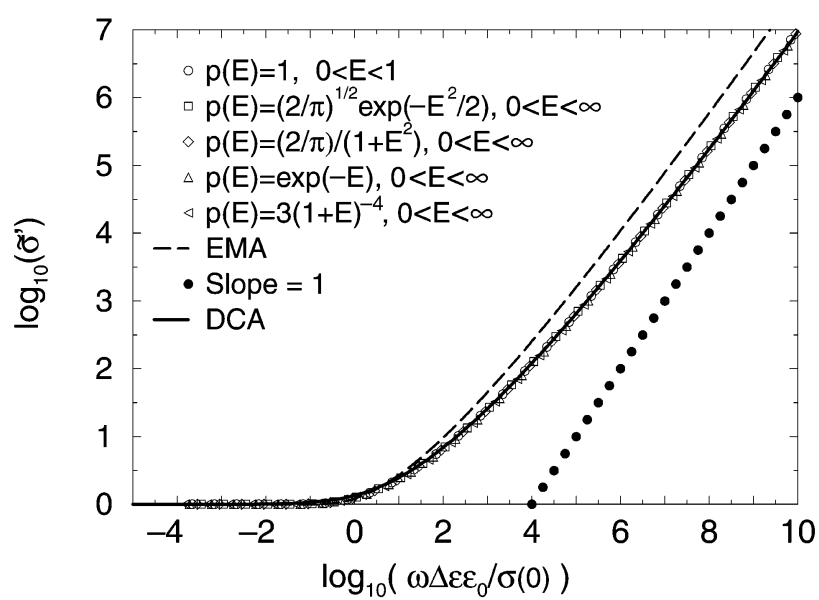

Fig. 7 Scaled real part of the frequency dependent conductivity for five different energy barrier probability distributions at large $\beta$, proving ac universality. The dashed line is the effective medium approximation (EMA), the full curve is the diffusion cluster approximation (DCA, empirically scaled as in Fig. 6b). Dots mark a line with slope unity.

approximation $[\ln \tilde{\sigma}=\mathrm{i} \tilde{\omega} / \tilde{\sigma}]^{4}$ (dashed curve). The latter is qualitatively correct, but quantitatively inaccurate.

\section{Some challenges for future research}

The random barrier model gives a good fit to many ac data. ${ }^{4}$ Interestingly, the experimental finding, that the ac conductivity in two dimensions is less frequency dependent than in three dimensions ${ }^{35}$ is reproduced by the random barrier model. ${ }^{36}$

Nevertheless, a number of important unsolved problems remain:

(i) How realistic is the model? It is known that the model may be derived by linearizing a hopping model with Fermi statistics and energy disorder, but how reliable is this linearization which itself is an approximation?

(ii) How can one explain the fact that the characteristic length for ionic glasses is found to be of order $1 \AA$ or even smaller, ${ }^{37}$ when the random barrier model predicts this length to be large at large disorder? In our opinion the most likely explanation is that ion conduction in glasses proceeds by a vacancy mechanism: ${ }^{38,39}$ If the effective charge carriers are few but mobile vacancies, the mean-square displacement per ion is small. Each vacancy would feel a random potential and consequently the hopping model would apply.

(iii) How are Coulomb interactions taken into account without making the model so complicated that it eludes analytical approximation?

(iv) What is the nature of the disorder? Does it have to be of a static nature or could it arise from the changing positions of the ions themselves (thus explaining the intriguing fact that some ionic crystals behave much like ionic glasses)? ${ }^{10}$

\section{Conclusion}

The random barrier model may be regarded as the ac conductivity analogue of the ideal gas model: Oversimplified, but still capturing the essential physics of the phenomenon. Experiments confirming model predictions do not tell us much about the disordered solid in question, only deviations from the model carry information about the conduction mechanism.

\section{References}

1 K. Funke, Prog. Solid State Chem., 1993, 22, 111.

2 S. R. Elliott, Solid State Ion., 1994, 70/71, 27.

3 A. K. Jonscher, Universal Relaxation Law, Chelsea Dielectric, London, 1996. 
4 J. C. Dyre and T. B. Schrøder, Rev. Mod. Phys., 2000, 72, 873.

5 J. O. Isard, J. Non-Cryst. Solids, 1970, 4, 357.

6 H. Namikawa, J. Non-Cryst. Solids, 1975, 18, 173.

7 A. K. Jonscher, Nature, 1977, 267, 673.

8 A. E. Owen, J. Non-Cryst. Solids, 1977, 25, 370.

9 A. Mansingh, Bull. Mater. Sci. (India), 1980, 2, 325.

10 K. Funke, Ber. Bunsen-Ges. Phys. Chem., 1991, 95, 955.

11 M. P. J. van Staveren, H. B. Brom and L. J. de Jongh, Phys. Rep., 1991, 208, 1

12 S. Summerfield, Philos. Mag. B, 1985, 52, 9.

13 K. Funke, B. Roling and M. Lange, Solid State Ionics, 1998, 105, 195.

14 A. R. Long, Adv. Phys., 1982, 31, 553.

15 S. Capaccioli, M. Lucchesi, P. A. Rolla and G. Ruggeri, J. Phys.: Condens. Matter, 1998, 10, 5595

16 J. C. Kimball and L. W. Adams, Jr., Phys. Rev. B, 1978, 18, 5851.

17 J. C. Dyre and T. B. Schrøder, Phys. Status Solidi B, 2002, 230, 5.

18 J. C. Dyre, Phys. Rev. B, 1993, 48, 12511.

19 P. N. Butcher, J. Phys. C, 1974, 7, 879.

20 H. Böttger and V. V. Bryksin, Hopping Conduction in Solids, Akademie, Berlin, 1985.

21 J. W. Haus and K. W. Kehr, Phys. Rep., 1987, 150, 263.

22 J.-P. Bouchaud and A. Georges, Phys. Rep., 1990, 195, 127.

23 D. L. Stein and C. M. Newman, Phys. Rev. E, 1995, 51, 5228.

24 N. G. van Kampen, Stochastic Processes in Physics and Chemistry, North-Holland, Amsterdam, 1981
25 H. Scher and M. Lax, Phys. Rev. B, 1973, 7, 4491; H. Scher and M. Lax, Phys. Rev. B, 1973, 7, 4502.

26 R. Kubo, J. Phys. Soc. Jpn., 1957, 12, 570.

27 J. C. Dyre, J. Appl. Phys., 1988, 64, 2456.

28 T. B. Schrøder, PhD Thesis, Roskilde University, 1999, condmat/0005127.

29 V. Ambegaokar, B. I. Halperin and J. S. Langer, Phys. Rev. B, $1971,4,2612$

30 (a) B. I. Shklovskii and A. L. Efros, Zh. Eksp. Teor. Fiz., 1971, 60, 867; (b) B. I. Shklovskii and A. L. Efros, Sov. Phys. JETP, 1971, 33, 468.

31 B. I. Shklovskii and A. L. Efros, Electronic Properties of Doped Semiconductors, Springer, Berlin, 1984.

32 P. Le Doussal, Phys. Rev. B, 1989, 39, 881 .

33 D. Stauffer and A. Aharony, Introduction to Percolation Theory, Taylor and Francis, London, 1992.

34 T. B. Schrøder and J. C. Dyre, Phys. Rev. Lett., 2000, 84, 310

35 D. L. Sidebottom, Phys. Rev. Lett., 1999, 83, 983.

36 J. C. Dyre and T. B. Schrøder, Phys. Rev. B, 1996, 54, 14884.

37 B. Roling, C. Martiny and K. Funke, J. Non-Cryst. Solids, 1999, 249, 201.

38 J. O. Isard, J. Non-Cryst. Solids, 1999, 246, 16.

39 (a) D. Wilmer, H. Feldmann and R. E. Lechner, Phys. Chem Chem. Phys., 2002, 4 (DOI: 10.1039/b201032a); (b) A. N. Cormack, J. Du and T. R. Zeitler, Phys. Chem. Chem. Phys., 2002, 4 (DOI: 10.1039/b201721k). 